\title{
RESEARCH
}

Open Access

\section{Relationship between the non-HDLc-to- HDLc ratio and carotid plaques in a high stroke risk population: a cross-sectional study in China}

Yan Liu ${ }^{1,2}$, Zhenwen Zhang ${ }^{2}$, Binlan Xia ${ }^{3}$, Liping Wang ${ }^{4}$, Hengzhong Zhang ${ }^{5}$, Yan Zhu ${ }^{2}$, Chao Liu ${ }^{1 *}$ (i) and Bin Song ${ }^{5^{*}}$

\begin{abstract}
Background: Evidence on the association between the non-high-density lipoprotein cholesterol (non-HDLc)-to-highdensity lipoprotein cholesterol (HDLc) ratio (non-HDLc/HDLc) and carotid plaques is still limited. This study aims to assess the relationship between the non-HDLc/HDLc and carotid plaques in a population with a high risk of stroke.

Methods: A cross-sectional study based on the community was conducted in Yangzhou, China. Residents (no younger than 40 years old) underwent questionnaire interviews, physical examinations, and laboratory testing during 20132014. The subjects with a high risk of stroke were further selected (at least three of eight risk factors including hypertension, atrial fibrillation, type 2 diabetes mellitus, dyslipidaemia, smoking, lack of exercise, overweight, and family history of stroke) or a transient ischaemic attack (TIA) or stroke history. Carotid ultrasonography was then performed on the high stroke risk participants. Carotid plaque was defined as a focal carotid intima-media thickness (cIMT) $\geq 1.5 \mathrm{~cm}$ or a discrete structure protruding into the arterial lumen at least $50 \%$ of the surrounding cIMT. Logistic regression was employed to evaluate the relationship between the non-HDLC/HDLc and carotid plaques.

(Continued on next page)
\end{abstract}

\footnotetext{
*Correspondence: liuchao@njucm.edu.cn; bsong1272@njmu.edu.cn

${ }^{1}$ Endocrine and Diabetes Center, Affiliated Hospital of Integrated Traditional

Chinese and Western Medicine, Nanjing University of Chinese Medicine, 8 Huadian East Road, Nanjing 210028, China

${ }^{5}$ Department of Center of Health Management, Clinical Medical College, Yangzhou University, 98 Nantong West Road, Yangzhou 225001, China

Full list of author information is available at the end of the article
}

(c) The Author(s). 2020 Open Access This article is licensed under a Creative Commons Attribution 4.0 International License, which permits use, sharing, adaptation, distribution and reproduction in any medium or format, as long as you give appropriate credit to the original author(s) and the source, provide a link to the Creative Commons licence, and indicate if changes were made. The images or other third party material in this article are included in the article's Creative Commons licence, unless indicated otherwise in a credit line to the material. If material is not included in the article's Creative Commons licence and your intended use is not permitted by statutory regulation or exceeds the permitted use, you will need to obtain permission directly from the copyright holder. To view a copy of this licence, visit http://creativecommons.org/licenses/by/4.0/. The Creative Commons Public Domain Dedication waiver (http://creativecommons.org/publicdomain/zero/1.0/) applies to the data made available in this article, unless otherwise stated in a credit line to the data. 
(Continued from previous page)

Results: Overall, 839 subjects with a high risk of stroke were ultimately included in the analysis, and carotid plaques were identified in 341 (40.6\%) of them. Participants in the highest non-HDLc/HDLc tertile group presented a higher proportion of carotid plaques than did those in the other two groups. After adjustment for other confounders, each unit increase in the non-HDLC/HDLc was significantly associated with carotid plaques (OR 1.55, 95\%Cl 1.28-1.88). In the subgroup analysis, the non-HDLc/HDLc was positively and significantly associated with the presence of carotid plaques in most subgroups. Additionally, the non-HDLc/HDLc interacted significantly with three stratification variables, including sex (OR 1.31 for males vs. OR 2.37 for females, $P$ interaction $=0.016)$, exercise (OR 1.18 for subjects without lack of exercise vs. OR 1.99 for subjects with lack of exercise, $P$ interaction $=0.004$ ) and heart diseases (OR 1.40 for subjects without heart diseases vs. OR 3.12 for subjects with heart diseases, $P$ interaction $=0.033$ ).

Conclusion: The non-HDLc/HDLc was positively associated with the presence of carotid plaques in a Chinese high stroke risk population. A prospective study or randomized clinical trial of lipid-lowering therapy in the Chinese population is needed to evaluate their causal relationship.

Keywords: Carotid plaques, Non-high-density lipoprotein cholesterol, High-density lipoprotein cholesterol, High stroke risk, Cross-sectional, Ratio, Atherosclerosis

\section{Background}

As one of the major atherosclerotic diseases, carotid atherosclerosis (CA) has become a leading cause of ischaemic stroke [1]. Reflecting the severity of lumen narrowing and irregular morphology of the carotid artery, carotid plaques, detected by non-invasive ultrasonography, could serve as a subclinical indicator of CA and provide early information to predict ischaemic stroke risk [2]. Therefore, it will be of great importance to prevent ischaemic stroke early by detecting and intervening in the risk factors for carotid plaques.

Previous investigations have indicated that the nonhigh-density lipoprotein cholesterol (non-HDLc)-tohigh-density lipoprotein cholesterol (HDLc) ratio (non-HDLc/HDLc) is significantly associated with arterial stiffness [3], non-alcoholic fatty liver diseases [4, 5], chronic kidney disease [6], diabetes [7], metabolic syndrome [8, 9] and insulin resistance [9]. Among these, some scholars found that the non-HDLc/HDLc was superior to either non-HDLc or HDLc alone [3, 5]. In addition, previous studies have investigated relationships between serum lipid profiles (including non-HDLc and HDLc) and CA [10-15]. One study found that the non-HDLc/HDLc was positively associated with the carotid intima-media thickness (cIMT) [14], and another study even indicated that postmenopausal females with a higher non-HDLc/HDLc were more likely to have carotid plaques [15]. However, the relationship between the non-HDLc/HDLc and carotid plaques is still largely unexplored in different populations.

Therefore, the present cross-sectional investigation was carried out in a population with a high stroke risk in China to elucidate whether the non-HDLc/HDLc is independently related to the presence of carotid plaques.

\section{Methods}

\section{Study population}

The Stroke Screening and Intervention Program (SSIP), a community-based, observational study implemented by the National Stroke Prevention Committee of China since 2012, was designed to evaluate and intervene in the stroke risk factors in Chinese adults. As a part of the SSIP, details of the study design and population used in the present cross-sectional study were reported elsewhere $[10,16]$. From among the initially recruited population, two communities (one from an urban location and the other from a rural location) were selected from November 2013 to March 2014 in Yangzhou (a city in eastern China), where most residents had lived for at least six months. A sample of 5529 inhabitants older than 40 years was randomly selected using stratified sampling by age and sex, based on the census results from the Chinese National Bureau of Statistics in 2010. In total, 5103 residents participated in this study, and complete baseline data were ultimately obtained from 4847 individuals.

High stroke risk participants were further selected to be analysed in this study, as only this population underwent carotid ultrasound measurement. The major inclusion criteria based on a holistic assessment of their risk factors for stroke were (1) hypertension, defined as elevated systolic blood pressure (SBP $\geq 140 \mathrm{mmHg}$ ) or diastolic blood pressure (DBP $\geq 90 \mathrm{mmHg})$ or use of antihypertensive drugs; (2) AF (atrial fibrillation), defined as an abnormal electrocardiogram suggesting atrial fibrillation; (3) type 2 diabetes mellitus, defined as elevated blood glucose (fasting $\geq 7.0 \mathrm{mmol} / \mathrm{L}$ or postprandial 2 $\mathrm{h} \geq 11.1 \mathrm{mmol} / \mathrm{L}$ or glycosylated haemoglobin $\geq 6.5 \%$ ), or treatment with oral antidiabetic medication or insulin; (4) dyslipidaemia, defined as triglyceride (TG) $\geq 1.70$ $\mathrm{mmol} / \mathrm{L}$ or total cholesterol $(\mathrm{TC}) \geq 5.20 \mathrm{mmol} / \mathrm{L}$ or low- 
density lipoprotein cholesterol (LDLc) $\geq 3.36 \mathrm{mmol} / \mathrm{L}$ or HDLc $\leq 0.90 \mathrm{mmol} / \mathrm{L}$ or current treatment with antilipidaemic medication; (5) smoking, defined as former history or current status of smoking; (6) lack of exercise, defined as physical exercise less than $30 \mathrm{~min}$ each time or less than three times a week or exercise duration less than one year (agricultural or industrial labour was regarded as no lack of exercise); (7) overweight, defined as body mass index (BMI) $\geq 26 \mathrm{~kg} / \mathrm{m}^{2}$; and (8) family history of stroke. Participants with at least three of the above eight risk features or history of a transient ischaemic attack (TIA) or stroke were considered members of the population with a high stroke risk. The major exclusion criteria were (1) pregnant woman or those who had recently delivered a child; (2) participants with renal insufficiency or other severe systemic illness; and (3) incomplete data collection without key parameters such as lipids or carotid plaques. The SSIP was conducted based on the Helsinki Declaration in 1975 and approved by the medical ethics committee of Jiangsu Subei People's Hospital (serial number: 2013KY-049). All participants signed written informed consent prior to any specimen or data collection.

\section{Data collection}

As previously described $[10,16]$, data on age, sex, region, education, employment status, smoking and exercise status, medical history and previous medication were collected by trained medical staff within a structured questionnaire. Physical examination was performed to measure height, weight, SBP and DBP. Overnight fasting blood samples were collected from each participant to measure fasting blood glucose (FBG), TG, TC, LDLc, HDLc, and homocysteine (HCY) levels with an AU6800 automatic chemistry analyser (Beckman Coulter, Brea, CA, USA) at the central laboratory of Jiangsu Subei People's Hospital. Normal reference ranges were 3.89-6.11 $\mathrm{mmol} / \mathrm{L}$ for $\mathrm{FBG}, 0.56-1.70 \mathrm{mmol} / \mathrm{L}$ for $\mathrm{TG}, 2.8-5.9$ $\mathrm{mmol} / \mathrm{L}$ for TC, $2.07-3.36 \mathrm{mmol} / \mathrm{L}$ for LDLc, $0.90-1.55$ $\mathrm{mmol} / \mathrm{L}$ for HDLc, and $0.0-15.0 \mu \mathrm{mol} / \mathrm{L}$ for $\mathrm{HCY}$. The value of non-HDLc was obtained by subtracting HDLc from TC [11], and elevated $\mathrm{HCY}$ was defined as > $15.0 \mu \mathrm{mol} / \mathrm{L}$.

\section{Carotid ultrasonography}

Carotid ultrasonography was conducted by certified sonographers using a portable instrument (LOGIC P5, 10.0 MHz, GE Healthcare Inc.; Boston, MA, USA). Carotid plaques were detected in three locations, including the common and internal carotid arteries and carotid bifurcation. Longitudinal and transverse scanning were performed on the far and near walls of these sections. The distance between the media-adventitia and lumen- intima boundaries was quantified at plaque-free sections of the carotid artery and defined as cIMT. Carotid plaque was defined as a focal cIMT $\geq 1.5 \mathrm{~cm}$ or a discrete structure protruding into the arterial lumen more than $50 \%$ from the surrounding cIMT, based on the American Society of Echocardiography [17].

\section{Statistical analysis}

Normally distributed continuous variables are described as the mean \pm standard deviation (SD) and were compared by one-way ANOVA, while non-normally distributed continuous variables are presented as the median (interquartile ranges) and were compared using the Kruskal-Wallis test. Categorical variables are summarized as counts with percentages and were compared using the chi-squared test or Fisher exact probability test. Subjects were also categorized into three groups in accordance with non-HDLc/HDLc tertiles. Logistic regression was employed to evaluate the correlation between the non-HDLc/HDLc (either as a continuous or categorical variable) and carotid plaques and calculate odds ratios (ORs) and 95\% confidence intervals (CIs). For multiple regression analysis, collinearity among independent variables was first assessed to ensure that it was appropriate to include them in the same model. If variance inflation factors were no less than 5 , collinearity was considered to exist, and such a variable was not included in the adjusted models. Finally, adjusted covariables included sex, age, region, education, employment status, BMI, SBP, DBP, FBG, TG, HCY, exercise and smoking status, family history of stroke, history of stroke and TIA, hypertension, type 2 diabetes, heart diseases and current antilipidaemic medication.

Subgroup analyses were performed based on some of the stroke risk factors and potential confounders mentioned above. For a continuous variable, it was first converted to a categorical variable according to the clinical or reference range cut-off point. Finally, subgroup analyses were conducted for the following variables: sex (male, female), age ( $<65, \geq 65$ years), region (urban, rural), overweight (yes/no), lack of exercise (yes/no), smoking (yes/ no), family history of stroke (yes/no), history of stroke (yes/no), heart diseases (yes/no), hypertension (yes/no), type 2 diabetes (yes/no), current antilipidaemic medication (yes/no), and high HCY (yes/no). The heterogeneity was also evaluated by incorporating two-factor interaction terms between the non-HDLc/HDLc (continuous) and included stratification variable, followed by the likelihood ratio test. All analyses were performed using Empower Stats (www.empowerstats.com, X\&Y solutions, Inc. Boston, MA, USA) and R software version 3.4.3 (http://www. r-project.org). Statistical significance was evaluated by a two-sided test with $P<0.05$. 


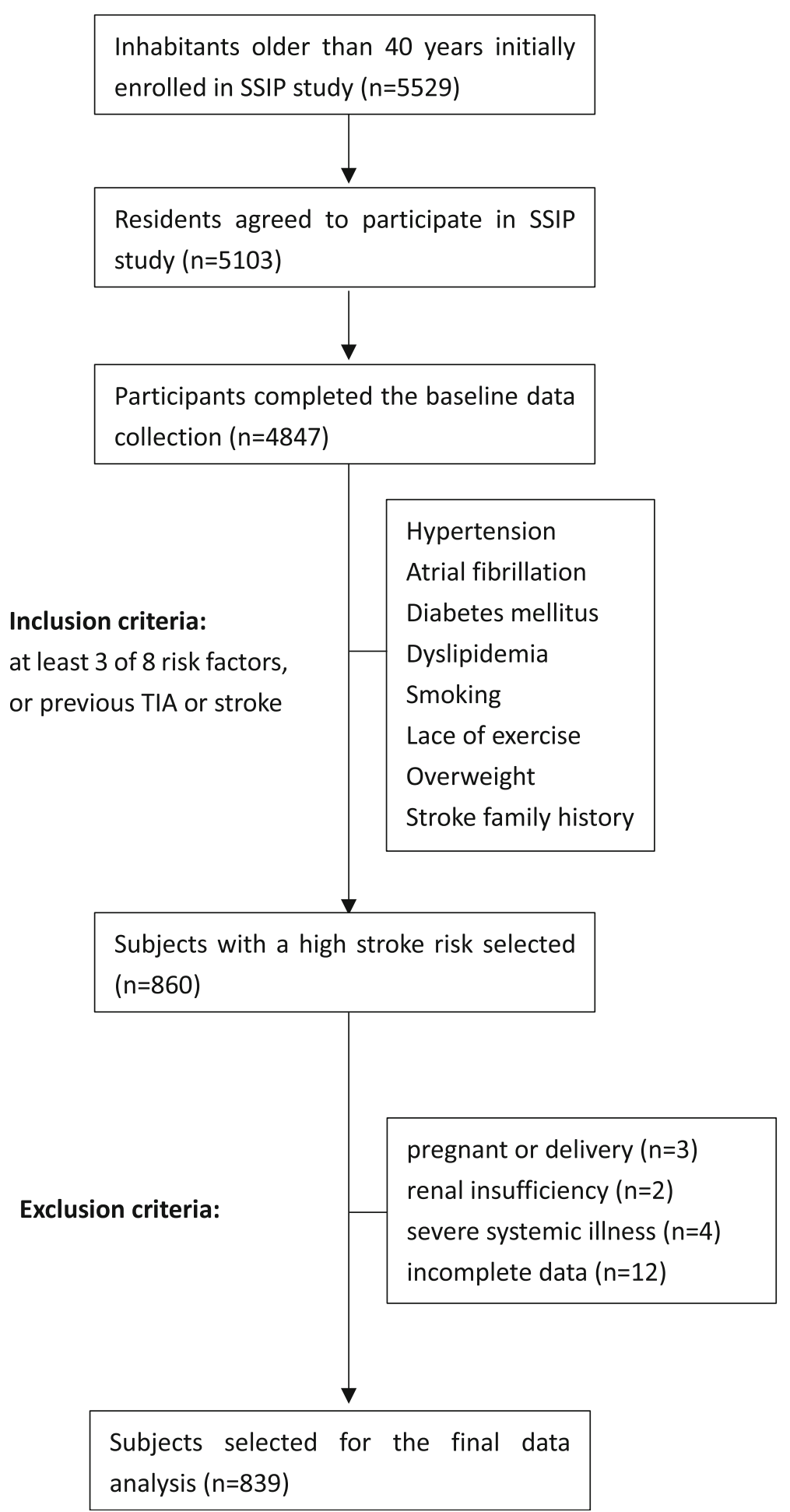

Fig. 1 Flowchart of participants selection

\section{Results}

Participants' baseline characteristics

For the data analysis, a total of 839 residents were ultimately enrolled in this study (Fig. 1 for a flow chart). The participants' baseline characteristics were shown in Table 1 according to tertiles of the non-HDLc/HDLc. Generally, all selected participants had an average age of $63.6 \pm 9.9$ years old. Males accounted for $54.2 \%$ of the 
Table 1 Baseline characteristics of participants

\begin{tabular}{|c|c|c|c|c|c|}
\hline \multirow[t]{2}{*}{ Characteristic } & \multicolumn{3}{|c|}{ non-HDLc/HDLc (tertile) } & \multirow[t]{2}{*}{$F / x^{2}$} & \multirow[t]{2}{*}{$P$} \\
\hline & $\begin{array}{l}\mathrm{T} 1 \\
(\boldsymbol{n}=287)\end{array}$ & $\begin{array}{l}\mathrm{T} 2 \\
(\boldsymbol{n}=286)\end{array}$ & $\begin{array}{l}\text { T3 } \\
(\boldsymbol{n}=266)\end{array}$ & & \\
\hline Male & $137(47.7)$ & $148(51.8)$ & $170(63.9)$ & 15.629 & $<0.001$ \\
\hline Age (years) & $64.8 \pm 10.0$ & $63.2 \pm 9.4$ & $62.7 \pm 10.2$ & 3.575 & 0.028 \\
\hline $\mathrm{BMI}\left(\mathrm{kg} / \mathrm{m}^{2}\right)$ & $25.54 \pm 3.63$ & $25.81 \pm 2.99$ & $26.05 \pm 3.20$ & 1.612 & 0.200 \\
\hline $\mathrm{SBP}(\mathrm{mmHg})$ & $140.08 \pm 15.55$ & $140.91 \pm 13.34$ & $139.80 \pm 16.01$ & 0.411 & 0.663 \\
\hline $\mathrm{DBP}(\mathrm{mmHg})$ & $86.87 \pm 9.17$ & $87.99 \pm 8.78$ & $87.38 \pm 9.62$ & 1.051 & 0.350 \\
\hline $\mathrm{FBG}(\mathrm{mmol} / \mathrm{L})$ & $6.27 \pm 1.87$ & $6.41 \pm 2.03$ & $6.84 \pm 2.48$ & 5.208 & 0.006 \\
\hline $\mathrm{TG}(\mathrm{mmol} / \mathrm{L})$ & $1.13(0.84-1.42)$ & $1.54(1.19-2.00)$ & $2.25(1.60-3.00)$ & 2.348 & $<0.001$ \\
\hline TC $(\mathrm{mmol} / \mathrm{L})$ & $4.27 \pm 0.82$ & $4.78 \pm 0.78$ & $5.22 \pm 0.92$ & 88.790 & $<0.001$ \\
\hline LDLc (mmol/L) & $2.14 \pm 0.58$ & $2.70 \pm 0.67$ & $2.93 \pm 0.81$ & 96.581 & $<0.001$ \\
\hline HDLc (mmol/L) & $1.56 \pm 0.35$ & $1.25 \pm 0.22$ & $1.03 \pm 0.19$ & 287.603 & $<0.001$ \\
\hline non-HDLc (mmol/L) & $2.71 \pm 0.64$ & $3.53 \pm 0.59$ & $4.19 \pm 0.78$ & 337.441 & $<0.001$ \\
\hline non-HDLC/HDLC & $1.78 \pm 0.41$ & $2.84 \pm 0.29$ & $4.10 \pm 0.61$ & 1810.918 & $<0.001$ \\
\hline $\mathrm{HCY}(\mu \mathrm{mol} / \mathrm{L})$ & $9.0(6.0-13.0)$ & $9.5(7.0-12.4)$ & $9.3(6.5-13.0)$ & 2.350 & 0.422 \\
\hline Urban & $179(62.4)$ & $147(51.4)$ & $102(38.4)$ & 31.908 & $<0.001$ \\
\hline Education & & & & 18.664 & 0.005 \\
\hline Primary school or under & $125(43.6)$ & $101(35.3)$ & $81(30.5)$ & & \\
\hline Middle or high school & $132(45.9)$ & $157(54.9)$ & $152(57.1)$ & & \\
\hline College or above & $14(4.9)$ & $18(6.3)$ & $26(9.8)$ & & \\
\hline Not reported & $16(5.6)$ & $10(3.5)$ & $7(2.6)$ & & \\
\hline Employment status & & & & 9.479 & 0.050 \\
\hline Unemployed & $81(28.2)$ & $95(33.2)$ & $107(40.2)$ & & \\
\hline Employed & $169(58.9)$ & $158(55.3)$ & $135(50.8)$ & & \\
\hline Not reported & $37(12.9)$ & $33(11.5)$ & $24(9.0)$ & & \\
\hline Overweight & $131(45.6)$ & $132(46.2)$ & $137(51.5)$ & 2.303 & 0.316 \\
\hline Smoking & 96 (33.5) & $117(40.9)$ & $129(48.5)$ & 12.948 & 0.002 \\
\hline Lack of exercise & $156(54.4)$ & $163(57.0)$ & $143(53.8)$ & 0.671 & 0.715 \\
\hline Family history of stroke & $60(20.9)$ & $62(21.7)$ & $54(20.3)$ & 0.159 & 0.924 \\
\hline History of stroke & $67(23.3)$ & $60(21.0)$ & $49(18.4)$ & 2.019 & 0.364 \\
\hline History of TIA & $13(4.5)$ & $17(5.9)$ & $9(3.4)$ & 2.053 & 0.358 \\
\hline Heart diseases & $79(27.5)$ & $65(22.7)$ & $60(22.6)$ & 2.447 & 0.294 \\
\hline Hypertension & $260(90.6)$ & $248(86.7)$ & $227(85.3)$ & 3.827 & 0.148 \\
\hline Type 2 diabetes & $81(28.2)$ & $83(29.0)$ & $92(34.6)$ & 3.092 & 0.213 \\
\hline Current antilipidaemic medication & $31(10.8)$ & $26(9.1)$ & $32(12.0)$ & 1.273 & 0.529 \\
\hline High HCY & $42(14.6)$ & $39(13.6)$ & $50(18.8)$ & 3.103 & 0.212 \\
\hline Carotid plaques & $108(37.6)$ & $108(37.8)$ & $125(47.0)$ & 6.509 & 0.039 \\
\hline
\end{tabular}

T1, non-HDLC/HDLc: 0.41-2.35; T2, non-HDLc/HDLc: 2.36-3.36; T3, non-HDLc/HDLc: 3.37-5.98. Continuous data are shown as the mean \pm standard deviation or median (interquartile), and categorical data are shown as $\mathrm{n}(\%)$. For categorical variables, $P$ value was calculated by $\mathrm{X} 2$ or Fisher's exact test. For continuous variables, $P$ value was calculated by one-way ANOVA or Kruskal-Wallis test

$B M I$ body mass index, SBP systolic pressure, DBP diastolic pressure, $F B G$ fasting blood glucose, TG triglyceride, $T C$ total cholesterol, $L D L C$ low-density lipoprotein cholesterol, HDLC high-density lipoprotein cholesterol, non-HDLC non-high-density lipoprotein cholesterol, HCY homocysteine

subjects, and carotid plaques were identified in 341 $(40.6 \%)$ of the 839 high stroke risk participants. The current sample size of 839 subjects with 341 cases had $80 \%$ power to detect at least an odds ratio of the non-
HDLc/HDLc on carotid plaques at a two-tailed $P$ value of $<0.05$. The average value of the non-HDLc/HDLc was $2.88 \pm 1.05$. Participants in the highest tertile group of the non-HDLc/HDLc (T3) were more likely to be 
male and had higher values of FBG, TG, TC, and LDLc, and higher education levels, and higher proportion of smoking and carotid plaques than those in the other groups. The opposite patterns were observed for age, HDLc and the proportion of urban regions. No statistically significant differences were detected in BMI, SBP, DBP, HCY, employment status, overweight, exercise status, family history of stroke, history of stroke and TIA, hypertension, type 2 diabetes, heart diseases, elevated $\mathrm{HCY}$ or current antilipidaemic medication among the different nonHDLc/HDLc groups (all $P$ values $>0.05$ ).

\section{Univariate analysis of carotid plaques}

By univariate binary logistic regression with carotid plaques as the dependent variable, shown in Table 2, it could be observed that male sex, age, SBP, DBP, FBG, TC, LDLc, nonHDLc, the non-HDLc/HDLc, HCY, rural region, smoking, history of stroke, hypertension, and elevated HCY were positively correlated with carotid plaques. In contrast, lack of exercise was negatively correlated with carotid plaques. Additionally, BMI, TG, HDLc, education, employment status, overweight, stroke family history, TIA, heart diseases, type 2 diabetes and current antilipidaemic medication were not significantly associated with carotid plaques.

\section{Associations of non-HDLc/HDLc and other crude lipid parameters with carotid plaques}

Three logistic regression models were constructed to evaluate the independent effects of the non-HDLc/HDLc on carotid plaques (Table 3). In the unadjusted model, the non-HDLc/HDLc, considered as a continuous variable (per unit increase), was significantly associated with the presence of carotid plaques (OR 1.23, 95\%CI 1.07$1.40)$. In the minimally adjusted model, as the nonHDLc/HDLc increased by one unit, the chance of having carotid plaques increased by $32 \%$ (OR 1.32, 95\%CI 1.131.53). In the fully adjusted model, for each additional unit of the non-HDLc/HDLc, the chance of having carotid plaques increased by $55 \%$ (OR 1.55 , 95\%CI $1.28-$ 1.88). For the purpose of sensitivity analysis, the continuous non-HDLc/HDLc was converted into a categorical variable (tertiles), and the $P$ for trend of the nonHDLc/HDLc with carotid plaques in the unadjusted or adjusted model was consistent with the results when the non-HDLc/HDLc served as a continuous variable.

Additionally, as shown in Table 4, in the fully adjusted model, continuous non-HDLc (OR 1.73, 95\%CI 1.42-2.11), TC (OR 1.57, 95\%CI 1.31-1.89) and LDLc (OR 1.71, 95\%CI 1.37-2.13) were significantly associated with carotid plaques, whereas neither HDLc or TG was not significantly associated with carotid plaques in either the unadjusted or adjusted models. In the mutually adjusted models
(Additional file 1), both of the non-HDLc/HDLc ratio (OR 1.30, 95\%CI 1.03-1.63) and non-HDLc (OR $1.80,95 \% \mathrm{CI} 1.10-2.95)$ were positively and significantly associated with the presence of carotid plaques even after adjusted with LDLc. However. LDLc was no longer significantly associated with carotid plaques when adjusted with non-HDLc (OR 0.94, 95\%CI 0.54-1.62).

\section{Associations of the non-HDLc/HDLc with carotid plaques in prespecified and exploratory subgroups}

Subgroup analyses explored possible associations of the non-HDLc/HDLc (treated as a continuous variable) with carotid plaques among different stratification variables (Fig. 2). The associations between the non-HDLc/HDLc and carotid plaques were similar among the following subgroups: age, region, overweight, smoking, family history of stroke, history of stroke, hypertension, type 2 diabetes, current antilipidaemic medication, and high HCY (all $P$ interaction $>0.05$ ). Noticeably, the non-HDLc/ HDLc interacted significantly with three stratification variables, including sex (OR 1.31, 95\%CI 1.02-1.68 for males vs. OR 2.37, 95\%CI 1.66-3.39 for females, $P$ interaction $=0.016)$, exercise $(\mathrm{OR} 1.18,95 \% \mathrm{CI} 0.89-1.55$ for subjects without lack of exercise vs. OR 1.99, 95\%CI 1.50-2.63 for subjects with lack of exercise, $P$ interaction $=0.004)$ and heart diseases (OR 1.40, 95\%CI 1.121.75 for subjects without heart diseases vs. OR 3.12, 95\%CI $1.88-5.20$ for subjects with heart diseases, $P$ interaction $=0.033$ ).

\section{Discussion}

In the present cross-sectional study based on the community population with a high risk of stroke in China, an independent, positive relationship was observed between the non-HDLc/HDLc - considered either as a continuous or categorical variable - and the presence of carotid plaques. Subjects had a 1.55-fold elevated chance of having carotid plaques with a per unit increase in the non-HDLc/HDLc after adjustment for other covariates, and this correlation was also found in most subgroups. The findings indicate that in a Chinese population with a high risk of stroke, an increased non-HDLc/HDLc is related to an elevated chance of having carotid plaques.

Non-HDLc represents atherogenic lipoproteins such as LDLc, very low-density lipoproteins, and small dense LDL (apolipoprotein B) [6], and the non-HDLc/HDLc ratio was detected to perform better than LDLc, HDLc or non-HDLc in estimating arterial stiffness [3] and non-alcoholic fatty liver disease [5]. However, there is limited evidence on the relationship between the nonHDLc/HDLc and carotid plaques. Qin et al. suggested that an increased non-HDLc/HDLc was significantly associated with elevated cIMT among Chinese individuals 
Table 2 Univariate analysis of carotid plaques

\begin{tabular}{|c|c|c|c|c|}
\hline Variables & Statistics & OR $(95 \% \mathrm{Cl})$ & $\beta$ & $P$ \\
\hline \multicolumn{5}{|l|}{ Sex } \\
\hline Female & $384(45.8)$ & Ref & & \\
\hline Male & $455(54.2)$ & $1.50(1.13,1.98)$ & 0.402 & 0.005 \\
\hline Age (years) & $63.6 \pm 9.9$ & $1.07(1.05,1.09)$ & 0.068 & $<0.001$ \\
\hline BMI $\left(\mathrm{kg} / \mathrm{m}^{2}\right)$ & $25.79 \pm 3.29$ & $0.97(0.93,1.01)$ & -0.033 & 0.132 \\
\hline $\mathrm{SBP}(\mathrm{mmHg})$ & $140.27 \pm 14.98$ & $1.02(1.01,1.03)$ & 0.021 & $<0.001$ \\
\hline $\mathrm{DBP}(\mathrm{mmHg})$ & $87.41 \pm 9.19$ & $1.02(1.00,1.03)$ & 0.016 & 0.033 \\
\hline FBG (mmol/L) & $6.50 \pm 2.15$ & $1.07(1.00,1.14)$ & 0.069 & 0.035 \\
\hline $\mathrm{TG}(\mathrm{mmol} / \mathrm{L})$ & $1.53(1.12-2.18)$ & $0.95(0.86,1.05)$ & -0.048 & 0.339 \\
\hline $\mathrm{TC}(\mathrm{mmol} / \mathrm{L})$ & $4.74 \pm 0.92$ & $1.33(1.14,1.54)$ & 0.282 & $<0.001$ \\
\hline LDLc (mmol/L) & $2.58 \pm 0.76$ & $1.45(1.20,1.75)$ & 0.371 & $<0.001$ \\
\hline HDLc (mmol/L) & $1.29 \pm 0.34$ & $0.93(0.62,1.40)$ & -0.072 & 0.730 \\
\hline non-HDLc (mmol/L) & $3.46 \pm 0.90$ & $1.36(1.16,1.59)$ & 0.306 & $<0.001$ \\
\hline non-HDLC/HDLC & $2.88 \pm 1.05$ & $1.23(1.07,1.40)$ & 0.204 & 0.003 \\
\hline $\mathrm{HCY}(\mu \mathrm{mol} / \mathrm{L})$ & $9.0(6.0-13.0)$ & $1.06(1.04,1.09)$ & 0.059 & $<0.001$ \\
\hline \multicolumn{5}{|l|}{ Region } \\
\hline Urban & $428(51.0)$ & Ref & & \\
\hline Rural & $411(49.0)$ & $1.46(1.10,1.92)$ & 0.376 & 0.008 \\
\hline \multicolumn{5}{|l|}{ Education } \\
\hline Primary school or under & $307(36.6)$ & Ref & & \\
\hline Middle or high school & $441(52.6)$ & $0.78(0.58,1.04)$ & -0.253 & 0.094 \\
\hline College and above & $58(6.9)$ & $1.11(0.63,1.95)$ & 0.104 & 0.717 \\
\hline Not reported & $33(3.9)$ & $0.73(0.35,1.53)$ & -0.317 & 0.403 \\
\hline \multicolumn{5}{|l|}{ Employment status } \\
\hline Unemployed & $283(33.7)$ & Ref & & \\
\hline Employed & $462(55.1)$ & $1.03(0.76,1.39)$ & 0.026 & 0.866 \\
\hline Not reported & $94(11.2)$ & $1.01(0.63,1.62)$ & 0.006 & 0.981 \\
\hline \multicolumn{5}{|l|}{ Overweight } \\
\hline No & $439(52.3)$ & Ref & & \\
\hline Yes & $400(47.7)$ & $0.83(0.63,1.09)$ & -0.190 & 0.178 \\
\hline \multicolumn{5}{|l|}{ Lack of exercise } \\
\hline No & $377(44.9)$ & Ref & & \\
\hline Yes & $462(55.1)$ & $0.70(0.53,0.93)$ & -0.355 & 0.012 \\
\hline \multicolumn{5}{|l|}{ Smoking } \\
\hline No & $497(59.2)$ & Ref & & \\
\hline Yes & $342(40.8)$ & $1.57(1.18,2.07)$ & 0.449 & 0.002 \\
\hline \multicolumn{5}{|l|}{ Family history of stroke } \\
\hline No & $663(79.0)$ & Ref & & \\
\hline Yes & $176(21.0)$ & $1.08(0.77,1.51)$ & 0.073 & 0.670 \\
\hline \multicolumn{5}{|l|}{ History of stroke } \\
\hline No & $663(79.0)$ & Ref & & \\
\hline Yes & $176(21.0)$ & $1.67(1.19,2.33)$ & 0.512 & 0.003 \\
\hline \multicolumn{5}{|l|}{ History of TIA } \\
\hline No & $800(95.4)$ & Ref & & \\
\hline
\end{tabular}


Table 2 Univariate analysis of carotid plaques (Continued)

\begin{tabular}{|c|c|c|c|c|}
\hline Variables & Statistics & OR $(95 \% \mathrm{Cl})$ & $\beta$ & $P$ \\
\hline Yes & $39(4.7)$ & $1.41(0.74,2.68)$ & 0.344 & 0.295 \\
\hline \multicolumn{5}{|c|}{ Heart diseases } \\
\hline No & $635(75.7)$ & Ref & & \\
\hline Yes & $204(24.3)$ & $1.21(0.88,1.66)$ & 0.189 & 0.246 \\
\hline \multicolumn{5}{|c|}{ Hypertension } \\
\hline No & $104(12.4)$ & Ref & & \\
\hline Yes & $735(87.6)$ & $2.13(1.34,3.38)$ & 0.755 & 0.001 \\
\hline \multicolumn{5}{|c|}{ Type 2 diabetes } \\
\hline No & $583(69.5)$ & Ref & & \\
\hline Yes & $256(30.5)$ & $1.23(0.91,1.66)$ & 0.207 & 0.172 \\
\hline \multicolumn{5}{|c|}{ Current antilipidaemic medication } \\
\hline No & $750(89.4)$ & Ref & & \\
\hline Yes & 89 (10.6) & $1.49(0.96,2.32)$ & 0.400 & 0.075 \\
\hline \multicolumn{5}{|l|}{ High HCY } \\
\hline No & $708(84.4)$ & Ref & & \\
\hline Yes & $131(15.6)$ & $2.40(1.64,3.51)$ & 0.875 & $<0.001$ \\
\hline
\end{tabular}

Continuous data are shown as the mean \pm standard deviation or median (interquartile), and categorical data are shown as $\mathrm{n}$ (\%)

$O R$ odds ratio, $\mathrm{Cl}$ confidence interval, $\beta$ regression coefficient, Ref reference

with metabolic syndrome [14]. The present study provides further evidence that the non-HDLc/HDLc was also positively correlated with the presence of carotid plaques. In addition, this study extends previous work on associations of the non-HDLc/HDLc with carotid plaques from postmenopausal middle-aged females [15] to the case of a high stoke risk population. Noticeably, the significant association between non-HDLc/HDLc with carotid plaques was mainly due to non-HDLc rather than HDLc, as HDLc did not show a significant influence in the present study. Furthermore, these findings from mutually adjusted models indicate that other components of non-HDLc beyond LDLc might have more contribution to carotid plaques than LDLc. In fact, previous studies have demonstrated that apolipoprotein B, but not LDLc, was associated with the progression of carotid plaques [18] or the future risk of coronary heart disease [19]. Therefore, the use of LDLc to assess cholesterol-related carotid plaques risk might underestimate future cardiovascular risk compared with small dense LDLc [18]. Although the concentration of small dense LDLc was not examined in this study, to a certain extent, the use of non-HDLc to assess carotid plaques appears to be superior to LDLc as non-HDLc components include small dense LDLc. Additionally, considering the non-significant effect of HDLc on carotid plaques in this study, the non-HDLc/HDLc ratio, as a clinically accessible indicator, might be more suitable to assess carotid plaques than LDLc.

In the univariate analysis, subjects with carotid plaques had higher values of age, SBP, DBP, FBG, and HCY and were more likely to be smokers, rural individuals and

Table 3 Relationships between the non-HDLc/HDLc and carotid plaques

\begin{tabular}{|c|c|c|c|c|c|c|c|c|}
\hline Variables & $\begin{array}{l}\text { Participant without } \\
\text { carotid plaque }(n, \%)\end{array}$ & $\begin{array}{l}\text { Participant with } \\
\text { carotid plaque }(n, \%)\end{array}$ & $\begin{array}{l}\text { Unadjusted OR } \\
(95 \% \mathrm{Cl})\end{array}$ & $P$ & $\begin{array}{l}\text { Minimally adjusted } \\
\text { OR (95\%Cl) }\end{array}$ & $P$ & $\begin{array}{l}\text { Fully adjusted } \\
\text { OR }(95 \% \mathrm{Cl})\end{array}$ & $P$ \\
\hline non-HDLC/HDLC & 498 & 341 & $1.23(1.07,1.40)$ & 0.003 & $1.32(1.13,1.53)$ & $<0.001$ & $1.55(1.28,1.88)$ & $<0.001$ \\
\hline \multicolumn{9}{|c|}{ non-HDLc/HDLc (tertile) } \\
\hline $\mathrm{T} 1$ & $179(35.9)$ & $108(31.7)$ & Ref & & Ref & & Ref & \\
\hline $\mathrm{T} 2$ & $178(35.7)$ & $108(31.7)$ & $1.01(0.72,1.41)$ & 0.974 & $1.12(0.78,1.62)$ & 0.546 & $1.18(0.81,1.73)$ & 0.393 \\
\hline T3 & $141(28.3)$ & $125(36.7)$ & $1.47(1.05,2.06)$ & 0.026 & $1.65(1.13,2.41)$ & 0.010 & $2.01(1.29,3.12)$ & 0.002 \\
\hline$P$ for trend & & & & 0.027 & & 0.010 & & 0.002 \\
\hline
\end{tabular}

Minimally adjusted OR controlled for sex, age, region, education, employment status, BMl, exercise and smoking status, and family history of stroke Fully adjusted OR controlled for sex, age, region, education, employment status, BMI, SBP, DBP, FBG, TG, HCY, exercise and smoking status, family history of stroke, history of stroke and TIA, hypertension, type 2 diabetes, heart diseases and current antilipidaemic medication

$O R$ odds ratio, $\mathrm{Cl}$ confidence interval, Ref reference 
Table 4 Relationships between the crude lipid parameters and carotid plaques

\begin{tabular}{lllllll}
\hline Variables & $\begin{array}{l}\text { Unadjusted } \\
\text { OR }(95 \% \mathrm{Cl})\end{array}$ & $\boldsymbol{P}$ & $\begin{array}{l}\text { Minimally adjusted } \\
\text { OR }(95 \% \mathrm{Cl})\end{array}$ & $\boldsymbol{P}$ & $\begin{array}{l}\text { Fully adjusted } \\
\text { OR (95\%Cl) }\end{array}$ \\
\hline non-HDLC (mmol/L) & $1.36(1.16,1.59)$ & $<0.001$ & $1.52(1.27,1.80)$ & $<0.001$ & $1.73(1.42,2.11)$ & $<$ \\
HDLC (mmol/L) & $0.93(0.62,1.40)$ & 0.730 & $0.90(0.57,1.40)$ & 0.634 & $0.90(0.55,1.47)$ & $0.675^{\mathrm{a}}$ \\
TC $(\mathrm{mmol} / \mathrm{L})$ & $1.33(1.14,1.54)$ & $<0.001$ & $1.45(1.23,1.71)$ & $<0.001$ & $1.57(1.31,1.89)$ & $<0.001^{\mathrm{a}}$ \\
LDLC $(\mathrm{mmol} / \mathrm{L})$ & $1.45(1.20,1.75)$ & $<0.001$ & $1.59(1.29,1.95)$ & $<0.001$ & $1.71(1.37,2.13)$ & $<0.001^{\mathrm{a}}$ \\
TG $(\mathrm{mmol} / \mathrm{L})$ & $0.95(0.86,1.05)$ & 0.339 & $0.99(0.90,1.10)$ & 0.863 & $0.97(0.87,1.09)$ & $0.662^{\mathrm{b}}$ \\
\hline
\end{tabular}

Minimally adjusted OR controlled for sex, age, region, education, employment status, BMI, exercise and smoking status, and family history of stroke Fully adjusted OR controlled for sex, age, region, education, employment status, BMI, SBP, DBP, FBG, HCY, exercise and smoking status, family history of stroke, history of stroke and TIA, hypertension, type 2 diabetes, heart diseases and current antilipidaemic medication plus TG ${ }^{a}$ or LDLC ${ }^{b}$

$O R$ odds ratio, $\mathrm{Cl}$ confidence interval, Ref reference

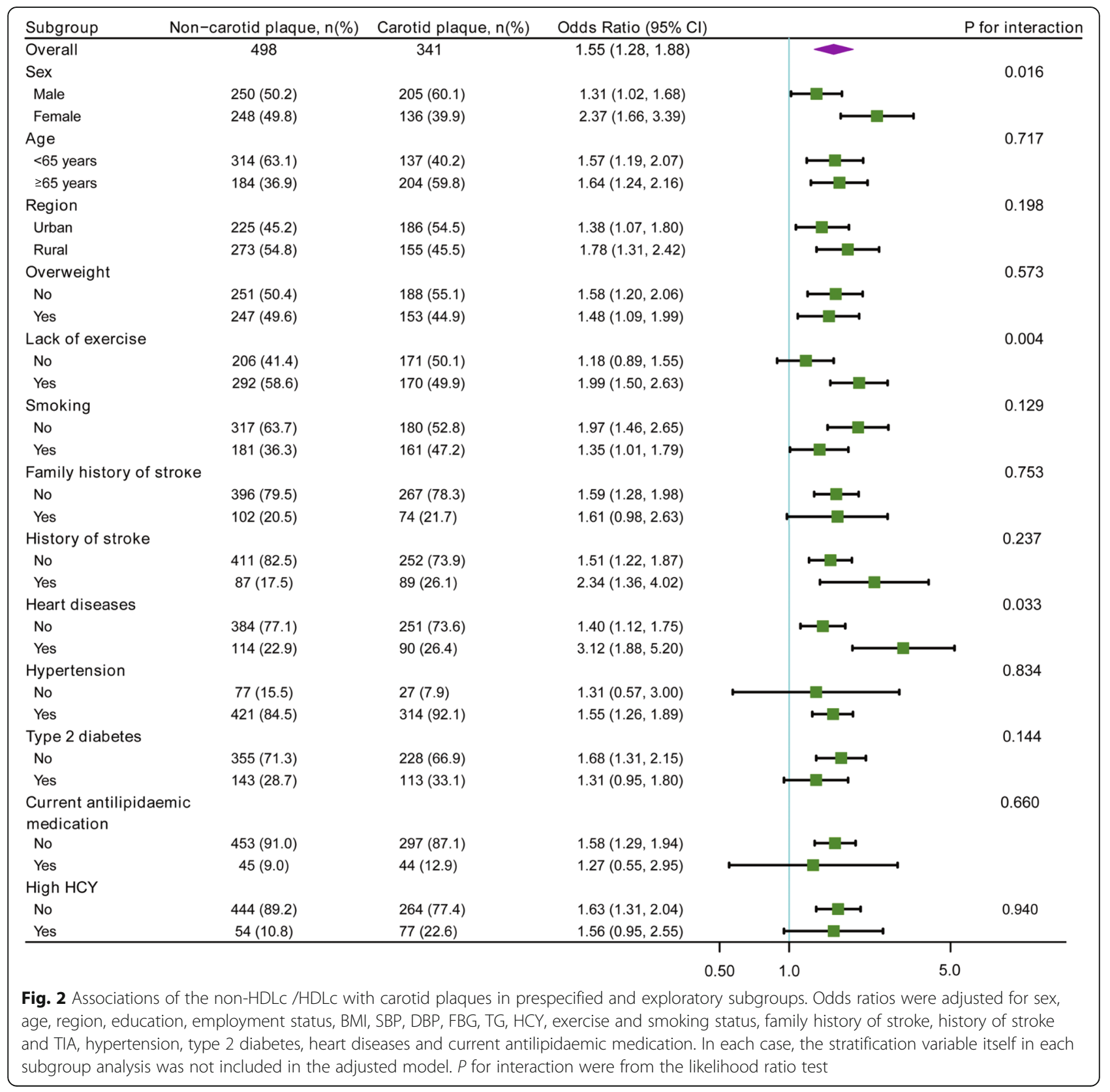


those with stroke history and hypertension, in agreement with the findings of previous studies [10,20,21]. Similar to the results in this study, Mi $\mathrm{T}$ et al. showed that men were more likely to suffer from carotid plaques in a high stroke risk population [10]. However, a higher chance of having carotid plaques was detected among women in a general population [22]. Additionally, the univariate analysis detected an unexpected phenomenon in which lack of exercise appeared to be a protective indicator against carotid plaques, which was inconsistent with the results of Mi T et al. [10]. Certainly, the protective effectiveness of lack of exercise disappeared in multiple analysis (data not shown). Nevertheless, the significant and positive association of the non-HDLc/HDLc with carotid plaques was stable after adjusting for as many potential confounders as possible, indicating that the non-HDLc/ HDLc might be independently associated with the presence of carotid plaques. Subgroup analysis is known to be helpful for understanding the trend of the nonHDLc/HDLc with carotid plaques in different populations. Some stroke risk factors and potential confounders were taken into account as stratification variables. The findings from this study showed that an elevated non-HDLc/HDLc was still significantly and independently related to a higher chance of having carotid plaques in most subgroups, providing further evidence that overall, the non-HDLc/HDLc is a reliable parameter for evaluating the presence of carotid plaques in this high stroke risk population. Subgroup analyses also showed evidence that sex, exercise status and heart diseases affected the observed correlation of the nonHDLc/HDLc with the presence of carotid plaques. Higher ORs have been observed in women and subjects with a lack of exercise or heart diseases, which might deserve further research.

Previous studies presented inconsistent conclusions regarding the relationship between traditional lipid profiles and the presence of carotid plaques in different populations. A community-based study from China suggested that TC and LDLc, but not TG, were strongly associated with the presence of $\mathrm{CA}$ in a general population [12]. However, several studies have previously reported that TG was also positively and significantly associated with $\mathrm{CA}$ in different populations [10, 23-25]. In particular, a large Chinese study found that TG was independently associated with carotid plaques in a high-risk stroke population, whereas LDLc and TC were not significantly associated with carotid plaques [10]. Conversely, findings from this study showed that LDLc and TC, but not TG or HDLc, were independently related to the presence of carotid plaques. Therefore, this issue is still controversial in the literature. The obvious differences between this and previous studies might be caused by discrepancies in the population selection or sample size. Additionally, the effect of TG or HDLc may have been masked by the existence of other powerful stroke risk factors in this high stroke risk population.

As an ongoing inflammatory process, atherosclerosis could affect multiple vascular territories including carotid and coronary arteries. The concomitant presence of carotid and coronary plaques is a frequently encountered clinical problem, and they appear to share common risk factors including dyslipidemia, diabetes, hypertension, smoking and obesity [26-28]. Recent studies showed a positive role of dual lipid-lowering treatment in coronary atherosclerosis regression, and the extent of coronary plaque regression was also positively associated with non-HDLc reduction [29]. Additionally, a cohort study indicated that plasma proprotein convertase subtilisin/kexin type 9 (PCSK9) levels were positively correlated with 10 -year progression of carotid atherosclerosis beyond LDLc [30]. These findings, when taken together with the results from this study, it would potentially implicative that dual lipidlowering therapy may be also beneficial for carotid atherosclerosis regression.

\section{Study strength and limitations}

This study has some strengths: (1) A strict statistical adjustment strategy has been used to minimize residual confounders, as this observational study is susceptible to potential confounding; (2) the target independent variable (non-HDLc/HDLc) was handled as either a continuous or categorical variable, which can reduce the contingency in the data analysis and enhance the robustness; (3) to make better use of data, effect modifier factor analysis was conducted and yielded stable conclusions in different subgroups in this study.

However, the findings of this study have several limitations. First, causal links between the non-HDLc/HDLc and carotid plaques cannot be established within a cross-sectional study design. Follow-up of these participants could help clarify this issue. Second, the subjects in this research are individuals with a high stroke risk. Therefore, universality or extrapolation is a certain deficiency in this study. The following study could extend this work to the general population. Third, although the multiple regression model adjusted for as many confounders as possible, residual confounding cannot be excluded due to the failure to collect other pivotal parameters, including apolipoprotein $\mathrm{B}$, the values of blood pressure during different days, the course and control of diabetes, and alcohol consumption. Therefore, collecting such key parameters could be essential in the future. Finally, given original design constraint, the composition and classification of carotid plaques were not further examined during data collection. In addition, ultrasound was used to evaluate the presence of plaque in this study, which may be less reliable in comparison 
with the high-resolution magnetic resonance imaging (MRI) [31]. However, ultrasound is safe, easily accessible, and inexpensive. Nevertheless, the use of MRI to evaluate the composition and types of plaques would have provided a significant add on the scientific value.

\section{Conclusions}

In the Chinese high stroke risk population based on the community, an elevated non-HDLc/HDLc was prominently correlated with the presence of carotid plaques. To assess the causal nature of the relationship, a prospective study or randomized clinical trial of lipid-lowering therapy is needed in the Chinese population.

\section{Supplementary information}

Supplementary information accompanies this paper at https://doi.org/10. 1186/s12944-020-01344-1.

\section{Additional file 1}

\section{Abbreviations}

SSIP: Stroke Screening and Intervention Program; BMI: Body mass index; CA: Carotid atherosclerosis; CIMT: Carotid intima-media thickness; AF: Atrial fibrillation; SBP: Systolic blood pressure; DBP: Diastolic blood pressure; FBG: Fasting blood glucose; TG: Triglyceride; TC: Total cholesterol; nonHDLC: Non-high-density lipoprotein cholesterol; LDLc: Low-density lipoprotein cholesterol; HDLc: High-density lipoprotein cholesterol; HCY: Homocysteine; TIA: Transient ischemic attack; PCSK9: Proprotein convertase subtilisin/kexin type 9; MRI: Magnetic resonance imaging; SD: Standard deviation; Ref: Reference; OR: Odds ratio; Cl: Confidence interval

\section{Acknowledgements}

We thank Xinglin Chen, PhD (Empower U, Department of Epidemiology and Biostatistics X\&Y solutions Inc. in Boston) and Jie Liu, PhD (Department of Vascular and Endovascular Surgery, Chinese PLA General Hospital) for their helpful review and comments regarding the manuscript.

\section{Authors' contributions}

$\mathrm{CL}$ and $\mathrm{HZ}$ took responsibility for the research questionnaire and the protocol for this study. BS, BX and LW conducted data collection and developed the original study design. $Y L, L W, Z Z$ and $Y Z$ analyzed the data together with the other authors; $\mathrm{YL}, \mathrm{BS}$ and $\mathrm{CL}$ interpreted the results; $\mathrm{YL}, \mathrm{ZZ}$ and BX wrote the article and the other authors revised it critically. All authors were responsible for the integrity of the data and the accuracy of the data analysis. The final version of the manuscript has been approved by all authors.

\section{Funding}

Design and data collection were supported by the Stroke Screening and Intervention Program (SSIP) implemented by the National Stroke Prevention Committee of China (2013); The development of the analytical methods and software used in this work was supported by the TCM Leading Talents Training Project of Jiangsu Province (SLJ0209) and the Ph.D startup foundation of Jiangsu Subei People's Hospital (BSQDJ0055). Writing and revising the manuscript were supported in part by the Cadre Health Project of Jiangsu Health Commission (BJ19011).

\section{Availability of data and materials}

The datasets used in the present study are available from the corresponding author on reasonable request.

\section{Ethics approval and consent to participate}

This study was performed based on the Helsinki Declaration in 1975 and approved by the medical ethics committee of Jiangsu Subei People's Hospital. All participants signed written informed consent.
Consent for publication

Not applicable.

\section{Competing interests}

The authors declare that they have no competing interests.

\section{Author details}

${ }^{1}$ Endocrine and Diabetes Center, Affiliated Hospital of Integrated Traditional Chinese and Western Medicine, Nanjing University of Chinese Medicine, 8 Huadian East Road, Nanjing 210028, China. ${ }^{2}$ Department of Endocrinology, Clinical Medical College, Yangzhou University, 98 Nantong West Road, Yangzhou 225001, China. ${ }^{3}$ Department of Ultrasonography, Clinical Medical College, Yangzhou University, 98 Nantong West Road, Yangzhou 225001, China. ${ }^{4}$ Department of Biobank, Clinical Medical College, Yangzhou University, 98 Nantong West Road, Yangzhou 225001, China. ${ }^{5}$ Department of Center of Health Management, Clinical Medical College, Yangzhou University, 98 Nantong West Road, Yangzhou 225001, China.

Received: 28 April 2020 Accepted: 6 July 2020

Published online: 13 July 2020

\section{References}

1. Baber U, Mehran R, Sartori S, Schoos MM, Sillesen H, Muntendam P, Garcia MJ, Gregson J, Pocock S, Falk E, Fuster V. Prevalence, impact, and predictive value of detecting subclinical coronary and carotid atherosclerosis in asymptomatic adults: the Biolmage study. J Am Coll Cardiol. 2015;65:1065-74.

2. Gupta A, Gialdini G, Giambrone AE, Lerario MP, Baradaran H, Navi BB, Marshall RS, ladecola C, Kamel H. Association between Nonstenosing carotid artery plaque on MR angiography and acute ischemic stroke. JACC Cardiovasc Imaging. 2016;9:1228-9.

3. Zhao W, Gong W, Wu N, Li Y, Ye K, Lu B, Zhang Z, Qu S, Li Y, Yang Y, Hu R. Association of lipid profiles and the ratios with arterial stiffness in middleaged and elderly Chinese. Lipids Health Dis. 2014;13:37.

4. Wang D, Wang L, Wang Z, Chen S, Ni Y, Jiang D. Higher non-HDLcholesterol to HDL-cholesterol ratio linked with increased nonalcoholic steatohepatitis. Lipids Health Dis. 2018;17:67.

5. Wang K, Shan S, Zheng H, Zhao X, Chen C, Liu C. Non-HDL-cholesterol to $\mathrm{HDL}$-cholesterol ratio is a better predictor of new-onset non-alcoholic fatty liver disease than non-HDL-cholesterol: a cohort study. Lipids Health Dis. 2018:17:196.

6. Zuo PY, Chen XL, Liu YW, Zhang R, He XX, Liu CY. Non-HDL-cholesterol to HDL-cholesterol ratio as an independent risk factor for the development of chronic kidney disease. Nutr Metab Cardiovasc Dis. 2015;25:582-7.

7. Zhang N, Hu X, Zhang Q, Bai P, Cai M, Zeng TS, Zhang JY, Tian SH, Min J, Huang HT, et al. Non-high-density lipoprotein cholesterol: high-density lipoprotein cholesterol ratio is an independent risk factor for diabetes mellitus: results from a population-based cohort study. J Diabetes. 2018;10: 708-14.

8. Reynoso-Villalpando GL, Sevillano-Collantes C, Valle Y, Moreno-Ruiz I, PadillaGutiérrez JR, Del Cañizo-Gómez FJ. ApoB/ApoA1 ratio and non-HDLcholesterol/HDL-cholesterol ratio are associated to metabolic syndrome in patients with type 2 diabetes mellitus subjects and to ischemic cardiomyopathy in diabetic women. Endocrinologia, diabetes y nutricion. 2019:66:502-11.

9. Kim SW, Jee JH, Kim HJ, Jin SM, Suh S, Bae JC, Kim SW, Chung JH, Min YK, Lee MS, et al. Non-HDL-cholesterol/HDL-cholesterol is a better predictor of metabolic syndrome and insulin resistance than apolipoprotein B/ apolipoprotein A1. Int J Cardiol. 2013;168:2678-83.

10. Mi T, Sun S, Zhang G, Carora Y, Du Y, Guo S, Cao M, Zhu Q, Wang Y, Sun $Q$, et al. Relationship between dyslipidemia and carotid plaques in a highstroke-risk population in Shandong Province, China. Brain Behav. 2016;6: e00610.

11. Wu J, Zhang J, Wang A, Chen S, Wu S, Zhao X. Association between nonhigh-density lipoprotein cholesterol levels and asymptomatic vulnerable carotid atherosclerotic plaques. Eur J Neurol. 2019;26:1433-8.

12. Hou Q, Li S, Gao Y, Tian H. Relations of lipid parameters, other variables with carotid intima-media thickness and plaque in the general Chinese adults: an observational study. Lipids Health Dis. 2018;17:107.

13. Ma H, Lin H, Hu Y, Li X, He W, Jin X, Gao J, Zhao N, Pan B, Gao X. Relationship between non-high-density lipoprotein cholesterol and carotid 
atherosclerosis in normotensive and euglycemic Chinese middle-aged and elderly adults. Lipids Health Dis. 2017;16:55.

14. Qin G, Tu J, Zhang C, Tang X, Luo L, Wu J, Liu L, Lu W, Tao L, Shen S, et al. The value of the apoB/apoAl ratio and the non-HDL-C/HDL-C ratio in predicting carotid atherosclerosis among Chinese individuals with metabolic syndrome: a cross-sectional study. Lipids Health Dis. 2015;14:24.

15. Masson W, Epstein T, Huerín M, Lobo M, Molinero G, Siniawski D. Association between non-HDL-C/HDL-C ratio and carotid atherosclerosis in postmenopausal middle-aged women. Climacteric. 2019;22:1-5.

16. Mi T, Sun S, Du Y, Guo S, Cong L, Cao M, Sun Q, Sun Y, Qu C. Differences in the distribution of risk factors for stroke among the high-risk population in urban and rural areas of Eastern China. Brain Behav. 2016;6:e00461.

17. Stein JH, Korcarz CE, Hurst RT, Lonn E, Kendall CB, Mohler ER, Najjar SS, Rembold CM, Post WS. American Society of Echocardiography carotid intima-media thickness task force: use of carotid ultrasound to identify subclinical vascular disease and evaluate cardiovascular disease risk: a consensus statement from the American Society of Echocardiography carotid intima-media thickness task force. Endorsed by the society for vascular medicine. J Am Soc Echocardiogr. 2008;21:93-111 quiz 189-190.

18. Qi Y, Liu J, Wang W, Wang M, Zhao F, Sun J, Liu J, Deng Q, Zhao D. High sdLDL cholesterol can be used to reclassify individuals with low cardiovascular risk for early intervention: findings from the Chinese multiprovincial cohort study. J Atheroscler Thromb. 2019;27:695-710.

19. Tsai MY, Steffen BT, Guan W, McClelland RL, Warnick R, McConnell J, Hoefner DM, Remaley AT. New automated assay of small dense lowdensity lipoprotein cholesterol identifies risk of coronary heart disease: the multi-ethnic study of atherosclerosis. Arterioscler Thromb Vasc Biol. 2014;34:196-201.

20. Zhao Q, Liu F, Wang YH, Lai HM, Zhao Q, Luo JY, Ma YT, Li XM, Yang YN LDL-C:HDL-C ratio and common carotid plaque in Xinjiang Uygur obese adults: a cross-sectional study. BMJ Open. 2018;8:e022757.

21. Yin JH, Song ZY, Shan PF, Xu J, Ye ZM, Xu XH, Zhang SZ, Liang Q, Zhao Y, Ren Z, Yu YP. Age- and gender-specific prevalence of carotid atherosclerosis and its association with metabolic syndrome in Hangzhou, China. Clin Endocrinol (Oxf). 2012;76:802-9.

22. Timóteo AT, Carmo MM, Ferreira RC. Carotid intima-media thickness and carotid plaques improves prediction of obstructive angiographic coronary artery disease in women. Angiology. 2013;64:57-63.

23. Masson W, Siniawski D, Lobo M, Molinero G, Huerín M. Association between triglyceride/HDL cholesterol ratio and carotid atherosclerosis in postmenopausal middle-aged women. Endocrinol Nutr. 2016;63:327-32.

24. Pacifico L, Bonci E, Andreoli G, Romaggioli S, Di Miscio R, Lombardo CV, Chiesa C. Association of serum triglyceride-to-HDL cholesterol ratio with carotid artery intima-media thickness, insulin resistance and nonalcoholic fatty liver disease in children and adolescents. Nutr Metab Cardiovasc Dis. 2014;24:737-43.

25. Touboul PJ, Labreuche J, Bruckert E, Schargrodsky H, Prati P, Tosetto A, Hernandez-Hernandez R, Woo KS, Silva H, Vicaut E, Amarenco P. HDL-C, triglycerides and carotid IMT: a meta-analysis of 21,000 patients with automated edge detection IMT measurement. Atherosclerosis. 2014;232:65-71.

26. Drakopoulou M, Oikonomou G, Soulaidopoulos S, Toutouzas K, Tousoulis D. Management of patients with concomitant coronary and carotid artery disease. Expert Rev Cardiovasc Ther. 2019;17:575-83.

27. Poi MJ, Echeverria A, Lin PH. Contemporary Management of Patients with concomitant coronary and carotid artery disease. World J Surg. 2018:42:272-82.

28. Steinvil A, Sadeh B, Arbel Y, Justo D, Belei A, Borenstein N, Banai S, Halkin A. Prevalence and predictors of concomitant carotid and coronary artery atherosclerotic disease. J Am Coll Cardiol. 2011;57:779-83.

29. Gragnano F, Calabrò P. Role of dual lipid-lowering therapy in coronary atherosclerosis regression: evidence from recent studies. Atherosclerosis. 2018:269:219-28.

30. Xie W, Liu J, Wang W, Wang M, Qi Y, Zhao F, Sun J, Liu J, Li Y, Zhao D. Association between plasma PCSK9 levels and 10-year progression of carotid atherosclerosis beyond LDL-C: a cohort study. Int J Cardiol. 2016;215:293-8.

31. Fabiani I, Palombo C, Caramella D, Nilsson J, De Caterina R. Imaging of the vulnerable carotid plaque: role of imaging techniques and a research agenda. Neurology. 2020;94:922-32.

\section{Publisher's Note}

Springer Nature remains neutral with regard to jurisdictional claims in published maps and institutional affiliations.

Ready to submit your research? Choose BMC and benefit from:

- fast, convenient online submission

- thorough peer review by experienced researchers in your field

- rapid publication on acceptance

- support for research data, including large and complex data types

- gold Open Access which fosters wider collaboration and increased citations

- maximum visibility for your research: over $100 \mathrm{M}$ website views per year

At BMC, research is always in progress.

Learn more biomedcentral.com/submissions 